J. Phys. IV France 138 (2006) 213-221

(C) EDP Sciences, Les Ulis

DOI: 10.1051/jp4:2006138024

\title{
Ablation laser pour la microélectronique plastique
}

\author{
A.-P. Alloncle ${ }^{1}$, B. Thomas ${ }^{1}$, D. Grojo ${ }^{1}$, Ph. Delaporte ${ }^{1}$, M. Sentis $^{1}$, \\ S. Sanaur ${ }^{2}, \mathrm{M}$. Barret ${ }^{2}$ et Ph. Collot ${ }^{2}$ \\ ${ }^{1}$ LP3 - UMR 6182 CNRS, Université de la Méditerranée, Campus de Luminy, \\ Case 917, 13288 Marseille Cedex 9, France \\ ${ }^{2}$ Centre de Microélectronique de Provence (CMP-GC), École des Mines de St. Étienne, \\ Département Packaging et Supports Souples (PS2), 13541 Gardanne, France
}

\begin{abstract}
Résumé. La microélectronique plastique connaît un développement sans précédent dans le domaine de la recherche. Cette étude s'intéresse à l'utilisation des lasers impulsionnels pour la réalisation de composants organiques sur supports souples. Les deux aspects plus particulièrement étudiés sont d'une part la gravure de polymère pour réaliser un canal entre la source et le drain, et d'autre part le développement d'un procédé de dépôt appelé LIFT pour Laser Induced Forward Transfer. Ce dernier pourrait notamment permettre de déposer des composés organiques non solubles.
\end{abstract}

\section{INTRODUCTION}

Les trois dernières années ont vu émerger un considérable regain d'intérêt pour les transistors à base de films minces de matériaux organiques (TFTs) en raison des possibilités remarquables d'applications de ces dispositifs en l'électronique plastique où la mise en oeuvre de procédés à bas coût, permettant des dépôts de grande surface, sur des supports flexibles, est impérative. Les deux applications majeures qui motivent ces recherches sont les étiquettes RFID et les écrans flexibles. Dans le premier cas, l'utilisation de polymères permettrait de diviser par 100 le coût de revient des étiquettes intelligentes qui pourraient alors remplacer les codes-barres des emballages. De même, des transistors organiques placés sur un support souple pourraient commander un écran à diodes organiques de type Oled. Deux techniques sont utilisées pour déposer ces composés organiques : l'évaporation sous vide, mais qui ne permet pas de réaliser de petites structures sans un système complexe de masquage, et le dépôt par jet d'encre qui n'est utilisable que pour les composés solubles. De plus, la résolution de ce dernier procédé n'est que de plusieurs dizaines de micromètres. L'utilisation de lasers impulsionnels pourrait permettre de développer de nouveaux procédés de fabrications de ces composés pour la microélectronique plastique.

Deux études seront présentées ci-après. La première a pour objectif de développer une méthode pour déposer localement et avec une bonne résolution spatiale des films minces, indépendamment de leur propriété de solubilité afin de fabriquer des transistors organiques. Cette technique dénommée LIFT pour 'Laser Induced Forward Transfer' repose sur l'éjection, sous l'effet de l'irradiation laser, d'un 'confetti' organique, à partir du substrat sur lequel le matériau a été préalablement déposé en film mince, vers un support cible maintenu à proximité. La seconde étude concerne la gravure de matériaux polymères préalablement déposés par jet d'encre sous forme de gouttes ou de lignes pour réaliser un canal entre la source et le drain des futurs transistors. La principale difficulté de cette dernière étude réside dans la fragilité du substrat composé de couches nanométriques qu'il ne faut pas endommager. Ces travaux sont donc basés sur les mécanismes d'ablation de matériaux polymères par laser impulsionnel. 


\section{DÉPÔT PAR LIFT}

\subsection{Principe}

Le procédé LIFT est basé sur l'irradiation et l'éjection, en une seule impulsion, d'une fine couche de matériau préalablement déposée sur un substrat transparent [1-4]. Les coefficients d'absorption respectifs de la couche et du substrat dépendent évidemment de la longueur d'onde du laser. En fonction de ces propriétés et de la fluence d'irradiation, les processus entrant en jeu lors de l'interaction diffèrent, allant d'une ablation thermique du matériau à une éjection mécanique sous l'action d'ondes de pression de forte intensité. Dans ce dernier cas, l'absorption du rayonnement est confinée entre le substrat et la couche de matériau à éjecter.On peut également réaliser le transfert de couches transparentes, en plaçant une fine couche de matériau absorbant entre le substrat et le film.

Durant les dernières années cette technique a été largement étudiée permettant le dépôt de métaux, d'oxydes métalliques, de semi-conducteurs et même de substances biologiques $[5,6]$ sur différentes sortes de substrats et à l'aide de différents types de laser.

Le procédé peut être décomposé en trois phases : ablation - transfert - et dépôt. Son contrôle passe par la connaissance des différents mécanismes se produisant lors de ces trois phases.

Le LIFT est donc une méthode très souple par rapport aux autres techniques de dépôt par laser. La PLD (Pulsed Laser Deposition) a été utilisée pour réaliser des films de pentacène [7]. C'est une technique basée sur l'évaporation de la couche qui permet un contrôle très précis de l'épaisseur déposée, mais avec laquelle il n'est pas possible de gérer la taille des dépôts. Pour éviter la décomposition chimique induite lors de l'évaporation, on peut utiliser la technique MAPLE (Matrix Assisted Pulsed Laser Evaporation) qui est similaire à la PLD mais dans laquelle le matériau est solubilisé avec un solvant puis congelé. Lors de l'irradiation de la cible, seul le solvant est vaporisé et le matériau se dépose sur une cible. Ce procédé ne permet pas non plus de contrôler les formes du dépôt et est plus complexe à mettre en œuvre. Il a cependant été utilisé avec succès pour réaliser des dépôts de polymères conjugués tels que du polythiophène [8]. De plus, ces techniques nécessitent que le dépôt se fasse sous vide ou sous très faible pression de gaz. Le MAPLE DW (direct writing) est un procédé plus proche du LIFT que des deux autres techniques. Tout comme le LIFT le matériau est déposé sur un substrat transparent, puis transféré par irradiation laser au travers du substrat sur une cible. Ce procédé nécessite cependant l'utilisation d'un solvant qui joue le rôle de matrice de transfert. Bien que relativement compliqué à mettre en œuvre, il a permis de réaliser des structures telles que des résistances de quelques centaines de micromètres et des capacités de dimensions millimétriques [9].

Les premiers travaux expérimentaux menés au laboratoire LP3 $[10,11]$ et dans d'autres groupes [12] ont mis en évidence que le procédé LIFT présentait des avantages très attractifs pour l'application considérée :

- le dépôt est réalisé à pression atmosphérique,

- la taille du dépôt est aisément contrôlée par la mise en forme du faisceau laser,

- c'est un phénomène mono-tir qui ne limitera pas la vitesse du procédé,

- il permet de réaliser des structures multicouches avec des résolutions micrométriques.

Les travaux présentés ci-après ont pour objectifs d'une part l'étude des mécanismes d'éjection d'un film PEDOT, (poly $(3,4)$ ethylenedioxythiophène) par ablation laser en fonction des conditions d'irradiation, et d'autre part la réalisation de dépôts de dimensions micrométriques de ce même matériau. Il s'agit d'un polymère conducteur avec lequel des transistors organiques ont récemment été fabriqués par des techniques d'impression de type jet d'encre [13].

\subsection{Dispositif expérimental}

La figure 2.1 présente le schéma de principe du dispositif expérimental. Les substrats sont irradiés par une seule impulsion laser. Un système optique simple permet d'obtenir une répartition quasi-uniforme 


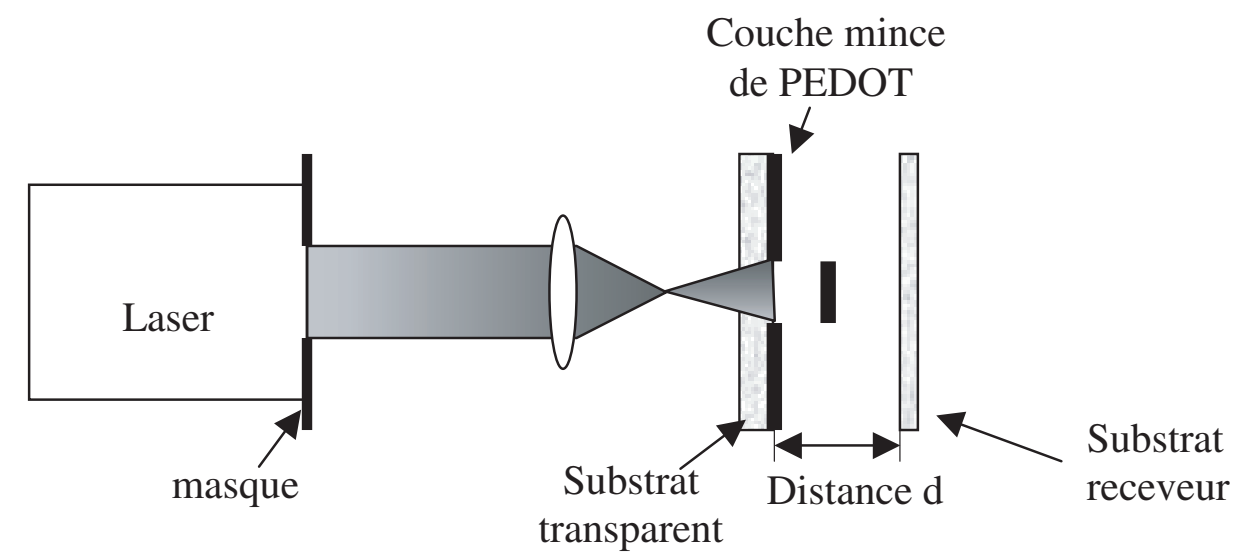

Figure 2.1. Schéma de principe du dispositif expérimental utilisé pour réaliser des dépôts par le procédé LIFT.

de l'énergie du faisceau sur la surface. Il comprend un masque métallique à ouverture carrée dont l'image est faite dans le plan de l'interface film-substrat au moyen d'une lentille de focale de $120 \mathrm{~mm}$. La surface de la zone irradiée est de $200 \mu \mathrm{m}$ x $200 \mu \mathrm{m}$. L'énergie de l'impulsion laser peut être modifiée par rotation d'une lame demi-onde placée sur le trajet du faisceau polarisé rectilignement. Un polariseur de Brewster assure l'ajustement de l'énergie du faisceau en réfléchissant la composante de la polarisation projetée selon le plan d'incidence (polarisation $\mathrm{P}$ ).

Le polymère est déposé par spin-coating sur des lames de verre d'une épaisseur de $1 \mathrm{~mm}$. Les films ainsi obtenus présentent une épaisseur uniforme de quelques centaines de nanomètres. Les expériences ont été effectuées avec laser Nd-YAG $\omega$ et $2 \omega$ émettant à $1064 \mathrm{~nm}$ et 532nm avec une durée d'impulsion de 7 ns.

Le substrat receveur est placé en contact avec la couche de polymère ou à une distance de $25 \mu \mathrm{m}$. Il est constitué soit d'une lame de verre de $1 \mathrm{~mm}$ d'épaisseur, soit d'un substrat souple, très mince, composé de plusieurs couches d'épaisseur nanométrique de matériaux différents.

\subsection{Résultats et discussions}

La première étape a consisté à étudier l'influence des longueurs d'ondes 1064nm et 532nm sur les processus d'ablation et de dépôt. Des images réalisées au microscope optique sont présentées sur les figures 2.2 et 2.3 .

A la longueur d'onde 532nm il est nécessaire d'irradier l'échantillon à des fluences élevées pour commencer à ablater le PEDOT. Les dépôts associés ne sont absolument pas homogènes, on retrouve le polymère fragmenté en petits morceaux dispersés sur le substrat receveur. A cette longueur d'onde la longueur d'absorption du PEDOT est de l'ordre de quelques microns. Une épaisseur de quelques centaines de nanomètre peut donc être considérée quasiment transparente. L'absorption du rayonnement s'effectue sur des défauts et/ou impuretés absorbant le rayonnement incident et provoquant des points chauds détruisant la couche de PEDOT.

Par contre à $1064 \mathrm{~nm}$, le polymère est beaucoup absorbant. L'ablation totale de la surface irradiée est réalisée à partir d'une fluence de $0.4 \mathrm{~J} / \mathrm{cm}^{2}$. Par contre, cette fluence n'est pas suffisante pour réaliser un dépôt homogène. Il est nécessaire d'irradier la couche de PEDOT avec une fluence de $1 \mathrm{~J} / \mathrm{cm}^{2}$ pour obtenir un transfert complet du polymère comme le montre les observations effectuées au microscope optique reportées figure 2.3. Dans ces conditions un spot de dimensions identiques à la zone ablatée est reconstitué avec une précision de 10 à $15 \mu \mathrm{m}$ (dimension du spot $200 \mu \mathrm{m}$ x $200 \mu \mathrm{m}$ ). 


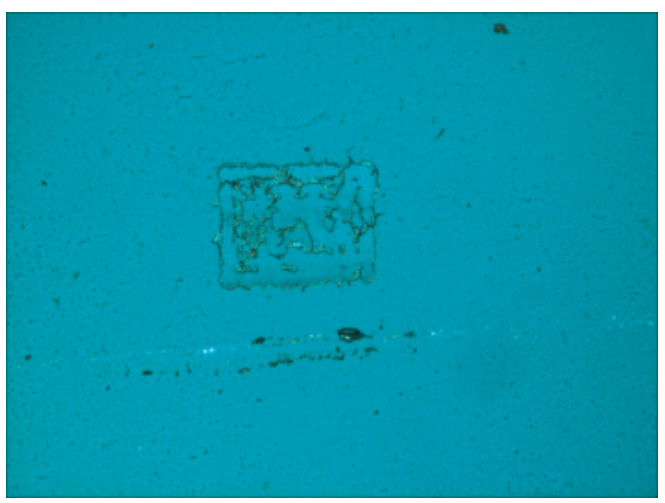

(a)

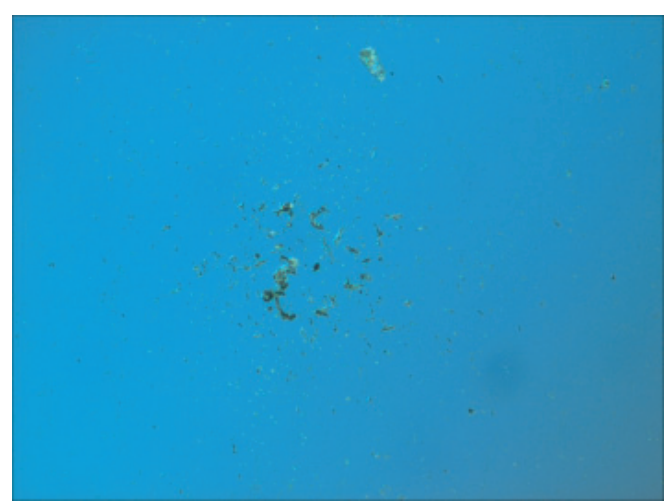

(b)

Figure 2.2. Transfert du PEDOT à $532 \mathrm{~nm}-1 \mathrm{~J} / \mathrm{cm}^{2}$ - a) zone ablatée, b) dépôt.

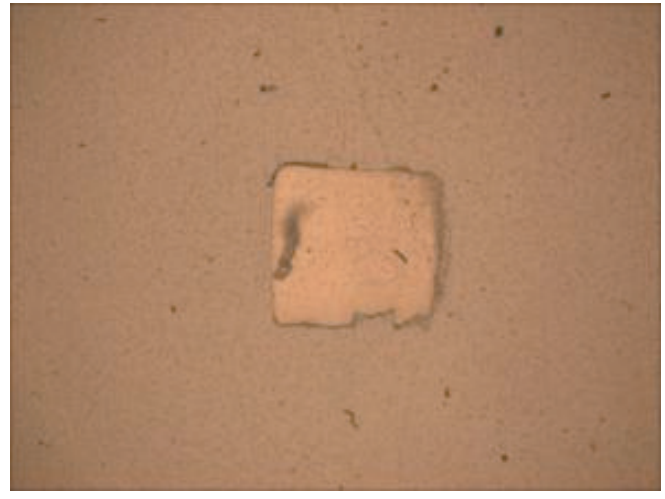

(a)

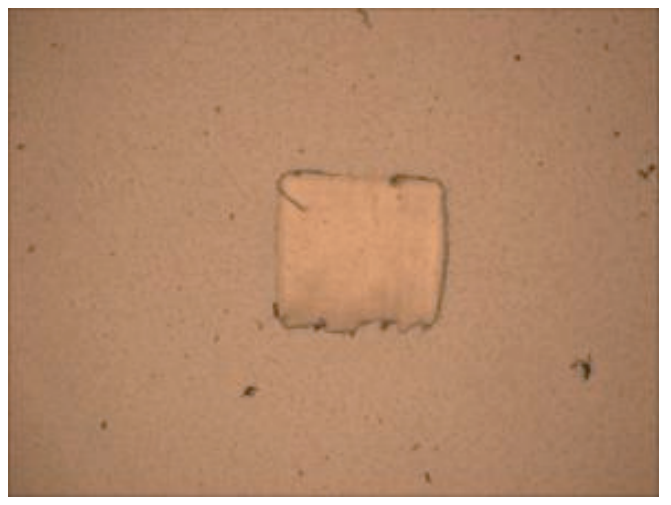

(c)

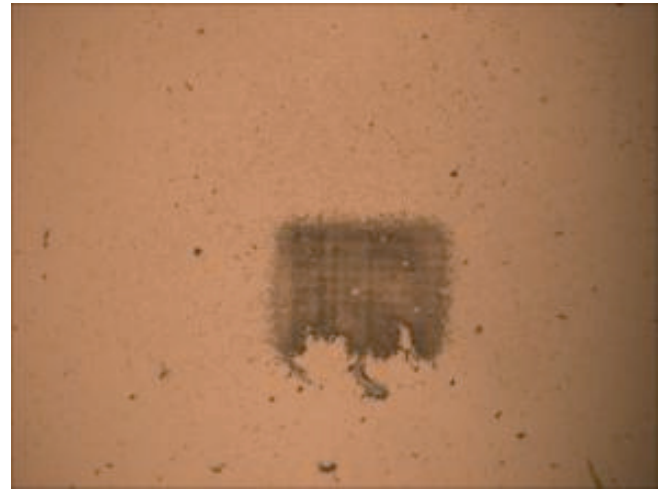

(b)

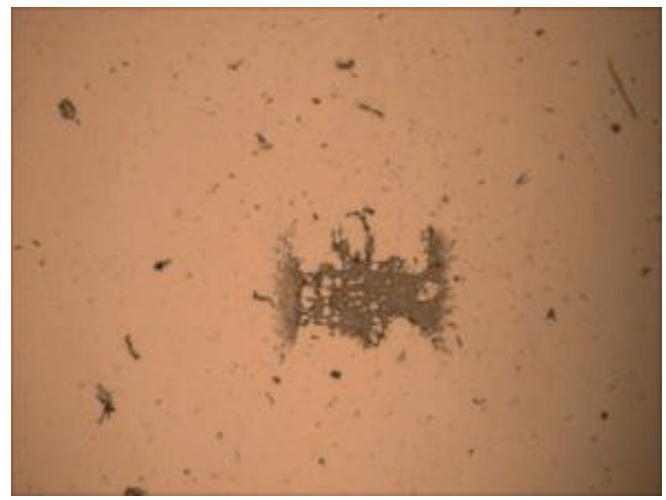

(d)

Figure 2.3. Transfert du PEDOT à $1064 \mathrm{~nm}$ - a) b) $0.9 \mathrm{~J} / \mathrm{cm}^{2}$ - a) zone ablatée, b) dépôt - c) d) $0.4 \mathrm{~J} / \mathrm{cm}^{2}-$ c) zone ablatée, d) dépôt. 


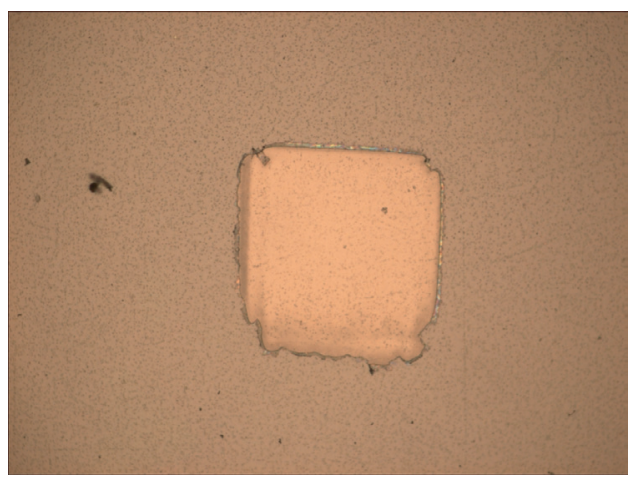

(a)

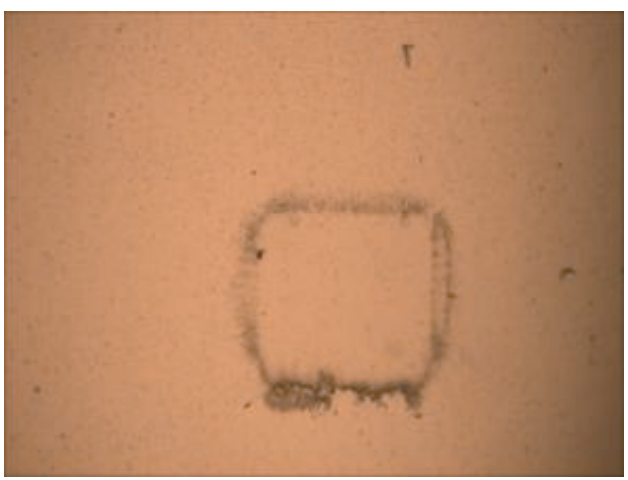

(b)

Figure 2.4. Transfert du PEDOT à $1064 \mathrm{~nm}-2.5 \mathrm{~J} / \mathrm{cm}^{2}$ - a) zone ablatée, b) dépôt.

Si l'on continue à augmenter la fluence le transfert est initié dans la première partie de l'impulsion mais l'énergie contenue dans le reste de l'impulsion contribue à complètement détruire la couche de polymère (figure 2.4). C'est une des raisons pour lesquelles, dans le cadre de la technique LIFT l'utilisation d'impulsion picoseconde ou sub-picoseconde présente un intérêt certain.

L'examen de la périphérie des zones ablatées montre que la couche est déchirée tout autour de la zone irradiée, conséquence de la génération d'ondes de pression de forte amplitude. L'absorption du rayonnement s'est effectuée dans le polymère, mais l'énergie déposée est restée confinée dans un petit volume défini par le substrat et la couche de polymère non perturbée par l'interaction. Il s'en suit une augmentation importante de température et de pression conduisant d'une part à des effets thermiques, d'autre part à des effets mécaniques conduisant à la rupture et à l'éjection de la couche. On constate que la surface visible du polymère déposé a noirci. Il est probable qu'il ait perdu ses propriétés. Des analyses complémentaires sont nécessaires pour contrôler la qualité du polymère transféré.

Des essais de LIFT du PEDOT sur substrat mince multicouches ont été réalisés afin d'en souligner les éventuelles difficultés. Les résultats sont présentés sur les figures 2.5 et 2.6 dans une configuration ou les substrats sont placés en contact (fig. 2.5) et une autre ou ils sont distants de $25 \mu \mathrm{m}$ (fig. 2.6). Le PEDOT est déposé de façon relativement homogène à des fluences identiques à celles

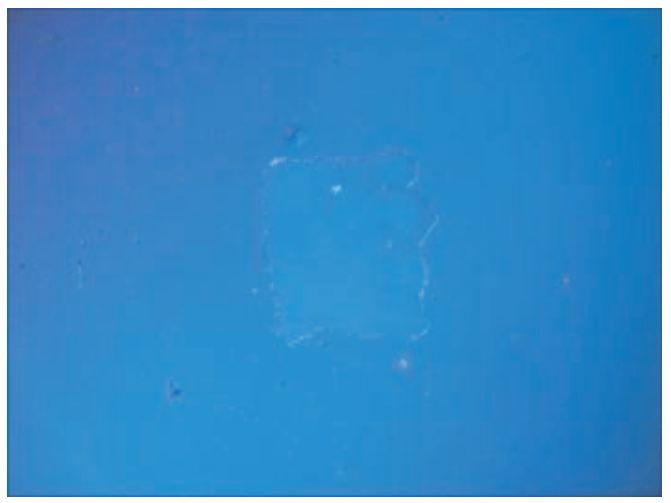

(a)

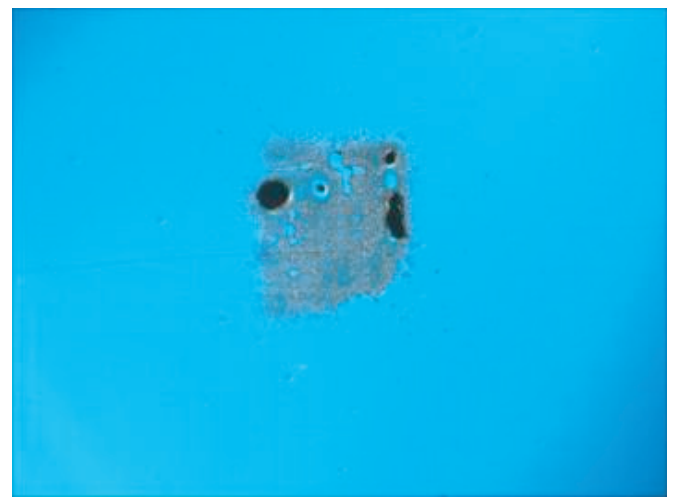

(b)

Figure 2.5. Transfert du PEDOT à $1064 \mathrm{~nm}$ sur substrat mince multi-couches placé en contact- $1 \mathrm{~J} / \mathrm{cm}^{2}-\mathrm{a}$ ) zone ablatée, b) dépôt. 


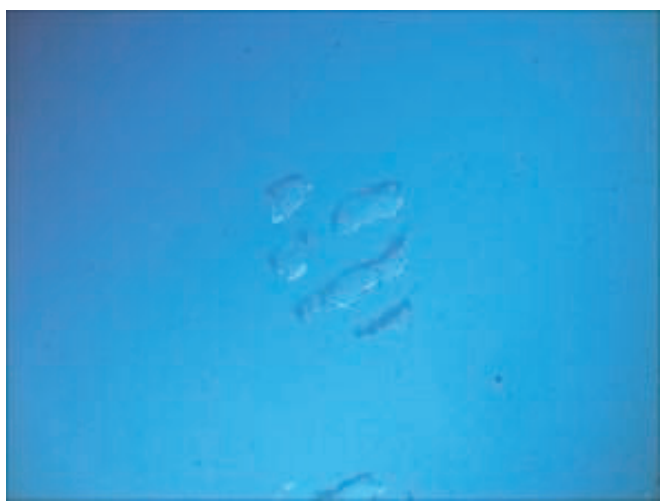

(a)

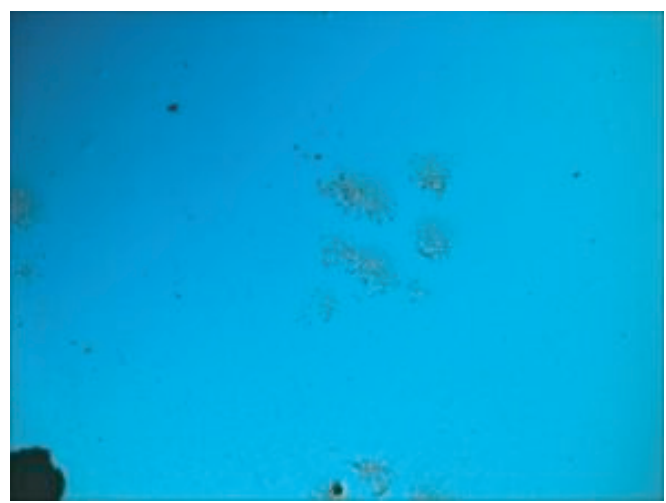

(b)

Figure 2.6. Transfert du PEDOT à $1064 \mathrm{~nm}$ sur substrat mince multi-couches distant de $\left.25 \mu \mathrm{m}-0.6 \mathrm{~J} / \mathrm{cm}^{2}-\mathrm{a}\right)$ zone ablatée, b) dépôt.

déterminées précédemment. Toutefois aux alentours de $1 \mathrm{~J} / \mathrm{cm}^{2}$ des dommages sont créés dans les substrats dans des zones de fluctuations d'énergie de la zone irradiée. Les seuils de dommages sont donc très proches des fluences optimales de transfert. Lorsqu'on éloigne le substrat receveur de quelques microns des interférences se construisent sur la couche de matériau à transférer entre l'onde incidente et celle réfléchie par le substrat. Elles détruisent l'homogénéité de l'ablation et donc du dépôt.

\section{GRAVURE DE POLYMÈRE}

\subsection{Dispositif expérimental}

Dans cette seconde partie le phénomène d'ablation laser est utilisé pour découper une ligne de PEDOT préalablement déposée par impression jet d'encre sur un substrat composé de 3 couches d'épaisseur nanométrique très fragiles et aux propriétés optiques différentes. Le but est d'ablater le PEDOT sans endommager le substrat. Pour mener l'étude des processus d'ablation en fonction de la longueur d'onde différentes sources laser ont été utilisées :

- un laser à excimères $\operatorname{ArF}(\lambda=193 \mathrm{~nm}$, Lambda Physik LPX220i) de durée d'impulsion 15ns,

- un laser $\operatorname{KrF}(\lambda=248 \mathrm{~nm}$, Lambda Physik EMG 203 MSC) de durée d'impulsion 27ns,

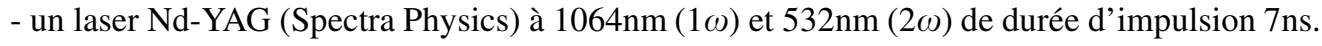

La mise en forme du faisceau est réalisée par un montage optique similaire à celui décrit précédemment (\$2.2). Dans le cas des lasers ultraviolets, l'énergie de l'impulsion est modifiée manuellement de manière continue à l'aide d'un atténuateur (Optec, AT4030).

\subsection{Résultats et discussions}

La première étape du projet est l'étude de l'influence de la longueur d'onde. Les figures 3.1 et 3.2 présentent les résultats obtenus lors de l'ablation par un laser $\mathrm{ArF}$ (fig 3.1) à 193nm et par un laser $\mathrm{KrF}$ (fig 3.2) à $248 \mathrm{~nm}$. On constate qu'un seul tir laser à $193 \mathrm{~nm}$ à une fluence de $20 \mathrm{~mJ} / \mathrm{cm}^{2}$ est suffisant pour détruire complètement le substrat alors que la ligne de PEDOT est intacte. Le substrat contient une couche polymère très absorbante à cette longueur d'onde et c'est probablement un mécanisme de photoablation qui est à l'origine de la destruction du substrat. Il n'y a d'ailleurs pas d'effets thermiques qui ont été observés à la périphérie de la zone ablatée. Les résultats obtenus avec un laser $\mathrm{KrF}$ sont très différents. Une irradiation réalisée avec une fluence plus élevée $\left(50 \mathrm{~mJ} / \mathrm{cm}^{2}\right)$ permet une ablation 


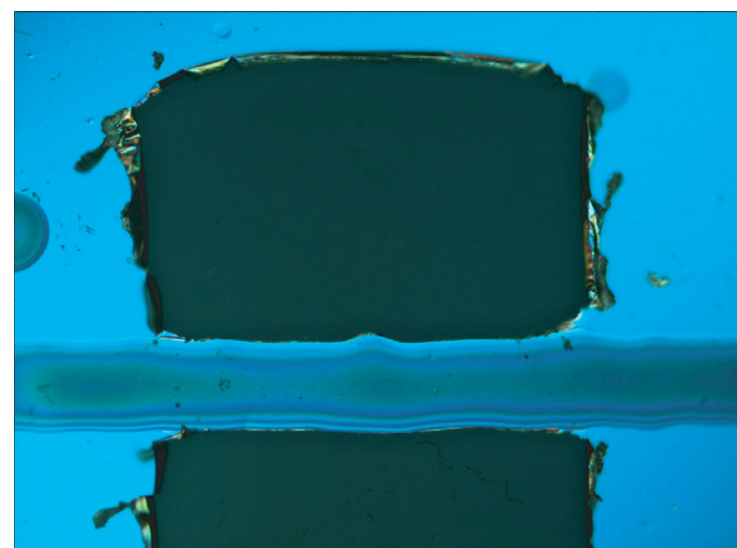

Figure 3.1. Irradiation d'une ligne de PEDOT déposée sur un substrat multicouche avec un laser $\operatorname{ArF}(193 \mathrm{~nm}, 1$ tir, $20 \mathrm{~mJ} / \mathrm{cm}^{2}$ ). La zone sombre correspond à la destruction du substrat par le faisceau. On observe que le PEDOT n'est pas modifié.

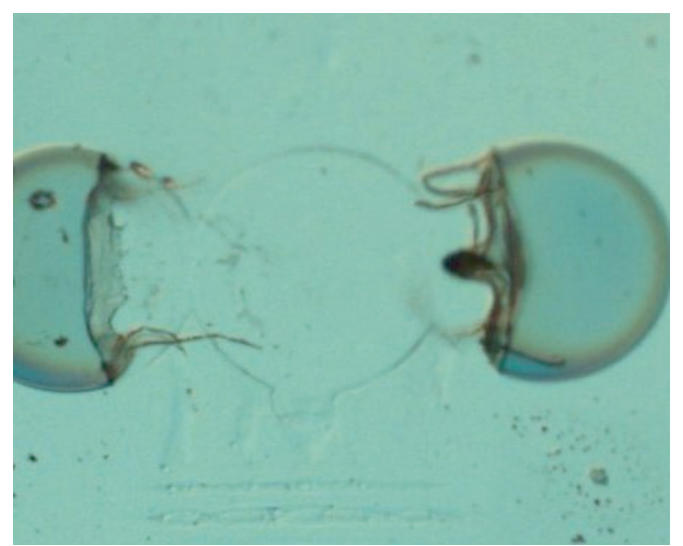

Figure 3.1. Irradiation d'une ligne de PEDOT déposée sur un substrat multicouche avec un laser $\mathrm{KrF}\left(248 \mathrm{~nm}, 1 \mathrm{tir}, 50 \mathrm{~mJ} / \mathrm{cm}^{2}\right)$.

relativement propre du PEDOT en un seul tir laser et engendre une légère modification du substrat. On constate également un noircissement sur les bords des gouttes de polymère découpées qui est significatif d'effets thermiques. L'utilisation de fluences plus faibles ne permet pas une ablation significative des lignes de polymère, surtout pour les plus épaisses.

D'autres essais ont été réalisés avec un laser Nd-YAG dans le visible (532nm) et le proche infrarouge $(1064 \mathrm{~nm})$. Les figures 3.3 et 3.4 présentent ces résultats. L'irradiation laser à 532nm permet de réaliser l'ablation des gouttes de PEDOT sans endommager le substrat pour des fluences proches de $100 \mathrm{~mJ} / \mathrm{cm}^{2}$. On constate tout d'abord que le mécanisme responsable de cette ablation est vraisemblablement d'origine thermique (figure 3.3 a). La diffusion thermique est cependant moins importante que lors

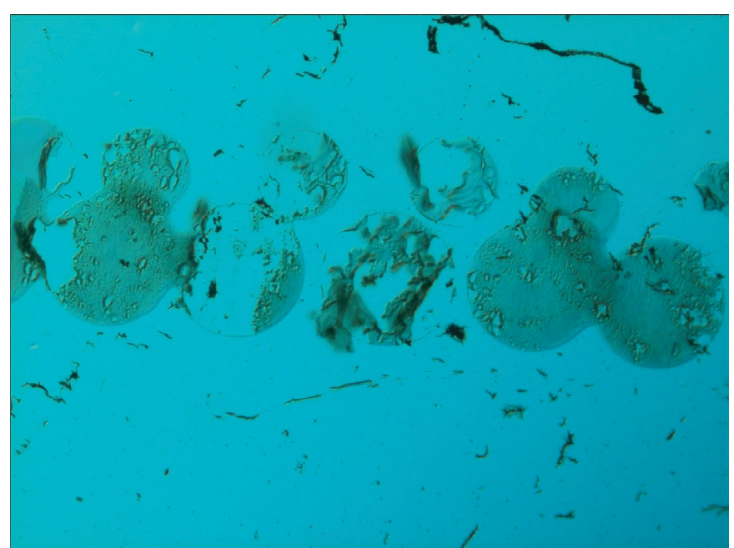

(a)

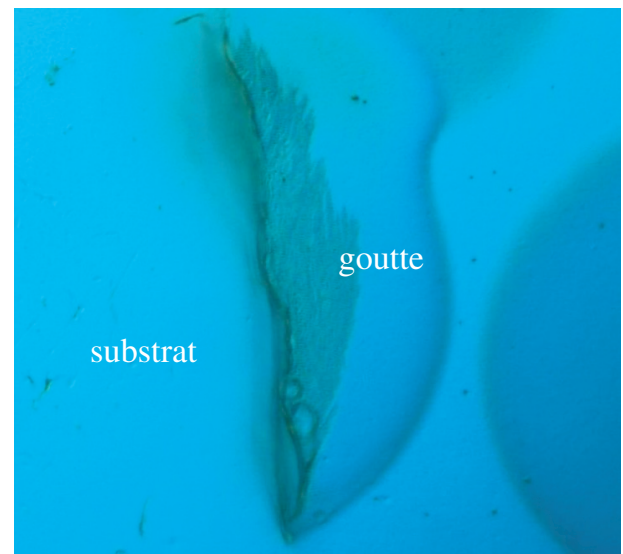

(b)

Figure 3.3. Ablation d'une ligne de PEDOT par un laser Nd-YAG à $532 \mathrm{~nm}\left(20 \mathrm{tirs}, 100 \mathrm{~mJ} / \mathrm{cm}^{2}\right)$. Les effets thermiques de l'ablation sont visibles sur certaines gouttes (a). Découpe d'une goutte de $50 \mu \mathrm{m}$ de diamètre sans endommagement du substrat (b). 


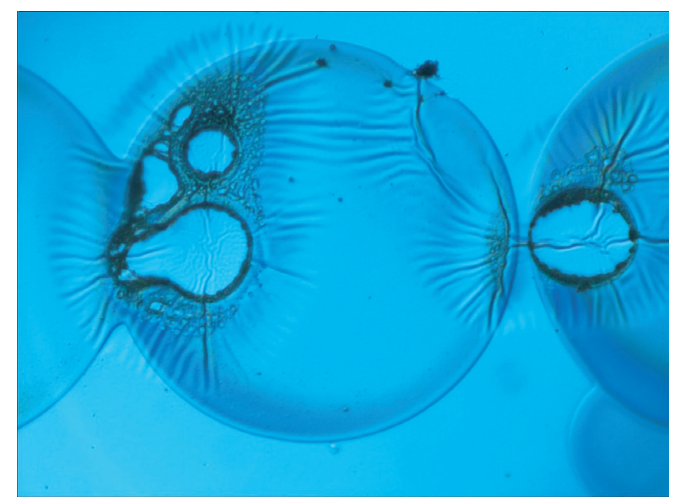

(a)

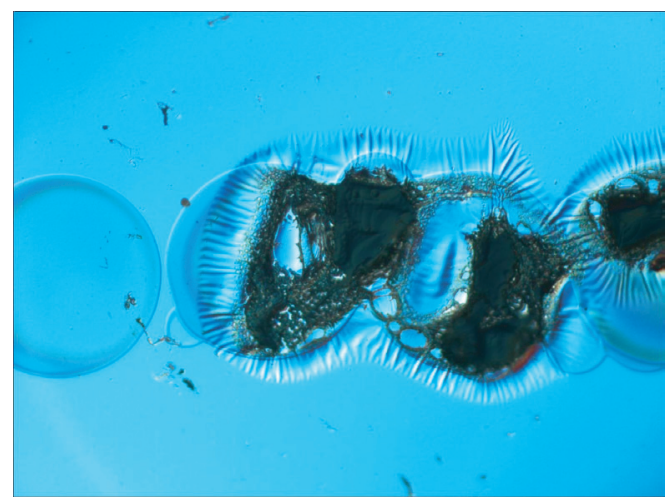

(b)

Figure 3.4. Ablation d'une ligne de PEDOT par un laser Nd-YAG à 1064nm. Les conditions d'irradiation étaient 1 tir, $460 \mathrm{~mJ} / \mathrm{cm}^{2}$ (a) et 10tirs, $1,2 \mathrm{~J} / \mathrm{cm}^{2}$. Les mécanismes thermiques de l'ablation du PEDOT et leurs influences sur le substrat sont clairement visibles sur ces images.

d'une irradiation à 248nm car les bords de la goutte découpée présentent moins d'effets thermiques résiduels et sont donc plus nets. Ceci peut être lié à la durée d'impulsion du laser Nd-YAG qui est de $7 \mathrm{~ns}$ au lieu de $27 \mathrm{~ns}$ pour le laser KrF. Les résultats des expériences menées avec un laser infrarouge mettent en évidence d'importants effets thermiques, à la fois sur le polymère à la périphérie des zones ablatées, mais également sur le substrat qui se plisse à cause de l'apparition d'un gradient de température trop important.

Ces résultats montrent que l'utilisation de longueurs d'onde courtes peut provoquer la destruction du substrat par effet photochimique et que des longueurs d'onde trop élevées génèrent des effets thermiques résiduels importants. Ces mécanismes sont liés aux coefficients d'absorption relatifs du PEDOT et des différents matériaux constituants le substrat multicouche. Un laser Nd-YAG doublé (532nm) devrait permettre de graver les gouttes de PEDOT avec une excellente résolution et de réaliser ainsi des largeurs de canal de l'ordre de la dizaine de micromètres.

\section{CONCLUSION}

Ces travaux s'inscrivent dans le cadre d'un programme destiné à déterminer l'intérêt d'utiliser des lasers impulsionnels pour permettre de développer de nouveaux procédés de fabrication de composés pour la microélectronique plastique. Deux procédés sont particulièrement étudiés : la gravure sélective et le LIFT (laser induced forward transfer).

Des essais de LIFT sur un polymère conducteur, le PEDOT ont été réalisés. Des spots de $200 \mu \mathrm{m}$ x $200 \mu \mathrm{m}$ ont été transférés avec une bonne précision. Toutefois des effets thermiques importants conduisent probablement à une détérioration des propriétés du matériau transféré. Deux objectifs sont envisagés dans la suite de ces études : d'une part l'utilisation de durée d'impulsions plus courtes (picoseconde et femtoseconde) et d'autre part l'utilisation d'une couche intermédiaire dont le rôle serait d'absorber le rayonnement et de restituer à la couche de PEDOT uniquement une énergie sous forme mécanique provoquant son éjection.

Les essais de gravure ont mis en évidence la très forte sensibilité du procédé à la longueur d'onde d'irradiation. Les seuils d'endommagement du substrat étudié sont très proches de ceux d'ablation du PEDOT. Le procédé nécessite donc une très bonne homogénéité de la répartition d'énergie dans la zone irradiée, ce qui risque de rendre son utilisation délicate dans le cadre du développement d'un procédé industriel. 


\section{Références}

[1] J. Bohandy, B.F. Kim and F.J. Adrian; Journal of Applied Physics, vol 60 (1986)

[2] I. Zergioti, D. Papazoglou, A. Karaiskou, C. Fotakis, E. Gamaly, A. Rode, Appl. Surf. Sci. 208209, p. 177-180 (2003)

[3] H. Yamada, T. Sano, T. Nagayama, I. Miyamoto, Appl. Surf. Sci. 197-198, p. 411-415 (2002)

[4] G. Dorcloman, P. Alloncle, O. Uteza, I. Mihailescu, Proceeding SPIE 5830, p. 276-280 (2005)

[5] P. Serra, J.M. Fernandez-Pradas, F.X. Berthet, M. Colina, J. Elvira, J.L. Morenza, Appl. Phys. A. 79 949-952 (2004)

[6] J.A. Barron, B.J. Spargo, B.R. Ringeisen, Appl. Phys. A. 79, 1027-1030 (2004)

[7] A. Salih, J. Marshall, J. Maud, J. Non-Cryst. Solids 230, p. 1240-1244 (1998)

[8] A. Gutiérrez-Llorente, G. Horowitz, R. Pérez-Casero, J. Perrière, J.L. Fave, A. Yassar, C. Sant, Org. Electronics 5, p. 29-34 (2004)

[9] D.B. Chrisey, A. Pique, J. Fitz-Gerald, R. Auyeung, R. McGill, H. Wu, M. Duignan, Appl. Surf. Sci. 154-155, p. 593-600 (2000)

[10] P. Alloncle, R. Bouffaron, J. Hermann, M. Sentis, Proceeding SPIE 6263 p6263R1-5 (2006)

[11] P. Alloncle, R. Bouffaron, J. Hermann, M. Sentis, Proceeding SPIE 6261 (2006)

[12] I. Zergioti, A. Karaiskou, D.G. Papazoglou, C. Fotakis, M. Kapsetaki, D. Kafetzopoulos, Appl. Surf. Sci. 247, p. 584-589 (2005)

[13] D. Zielke, A. Hübler, U. Hahn, N. Brandt, M. Bartzsch, U. Fügmann, T. Fischer, J. Veres, S. Ogier, Appl. Phys. Lett. 87, 123508 (2005) 